\title{
ON MODULAR FORMS OF DIMENSION -2
}

\author{
JOHN RODERICK SMART $\left(^{1}\right)$
}

1. This paper is devoted to the problem of obtaining modular forms of dimension -2 (see $\$ 2$ for definitions) by summing conditionally convergent Poincaré series (3.1) in a particular fashion. Rademacher [23] initiated the method when he recovered the functional equation of the modular invariant $J(\tau)$ from its Fourier series. Lehner [10] made further developments and applied the method to the case of forms of negative dimension on the full modular group. We consider the problem for subgroups of finite index in the modular group. The essential device is a lemma which allows us to rearrange certain conditionally convergent series. This has come to be known as the Rademacher Lemma. In our case we need a lemma which is considerably more general than that used by either Rademacher or Lehner. This is due to the fact that a subgroup of the modular group may have many generators. Also important in this regard is the fact that a fundamental region may have finite cusps at which the functions must be shown to have the proper behavior. Knopp ([7], [8]) has given several versions of the Rademacher Lemma which parallel ours. He applies it to the problem of obtaining forms of positive dimension rather than forms of negative dimension; for that reason the summands in the conditionally convergent series are different. This method requires a nontrivial estimate of certain Kloosterman sums.

There has been another approach to this problem. Hecke [4] introduced a convergence factor and then by a limiting operation obtained forms of dimension - 2. Petersson ([19], [22]) has developed this method to apply not only to the modular group but also to other discontinuous groups as well. For this method the estimation of the Kloosterman sums does not arise.

In $\S 2$ we give introductory material. In $\S 3$ we define the Poincare series and show that they converge conditionally to functions holomorphic in the upper half plane. Lemma 1 contains two different methods of summing these series. $\$ 4$ is devoted to the proof of the fundamental Rademacher Lemma. At the end of this section, in Lemma 3, we show that our Poincare series can be summed in a different (and more important) way. The main theorem appears in $\$ 5$. In this section the functional equations (2.4) are

\footnotetext{
Presented to the Society, January 23, 1961 under the title On modular forms of dimension - 2 for subgroups of the modular group; received by the editurs March 23, 1964.

(1) This paper is essentially my doctoral dissertation written under the direction of Joseph Lehner and submitted at Michigan State University.
} 
shown to be satisfied. Furthermore, we show that the functions defined by these serie: have the proper local variable expansion at the finite cusps. Kloosterman sums are considered in $\$ 6$. We give several examples when the Kloosterman sums $(6.1)$ have the estimate $O\left(|c|^{1 / 2+c}\right)$. Our results are based on a theorem of Petersson [21] as to when certain congruence subgroups and multiplier systems will give rise to Kloosterman sums with nontrivial estimate. In $\$ 7$ we sketch a proof of the "inner product formula" (7.2) for the Poincare series with which we deal. This along with some recent results of Lewittes [13] concerning the Weierstrass gap sequence at the infinite cusp determines a basis of the cusp forms of dimension - 2 for the principal congruence subgroups of levels 7, 8, 9, 10 and 12 in terms of our Poincaré series. In other words, these results determine a basis for the abelian differentials on the Riemann surface associated with these principal congruence subgroups.

2. The homogeneous modular group $\bar{\Gamma}(1)$ is the set of all two-by-two matrices of determinant 1 with rational integral entries. To each $V$ $=(a b \mid c d) \in \bar{\Gamma}(1)$ corresponds a linear fractional transformation

$$
V z=V(z)=(a z+b) /(c z+d)
$$

which maps the complex upper half plane $\mathscr{E}=\{z: \operatorname{Im} z>0\}$ onto itself. Note that $V$ and $-V$ correspond to the same transformation $V z$. The group of transformations is denoted by $\Gamma(1)$ and is known to be generated by

$$
S z=-1 / z, \quad U z=z+1 .
$$

The homogeneous principal congruence subgroup of lever $N, \bar{\Gamma}(N)$, consists of those elements $V=(a b \mid c d) \in \bar{\Gamma}(1)$ which satisfy $V \equiv \pm I \bmod N$ where $I=\left(\begin{array}{llll}1 & 0 & 0 & 1\end{array}\right)$ and we mean elementwise congruence. Let $\Gamma(N)$ denote the corresponding group of substitutions. We let $\Gamma$ denote a subgroup of $\Gamma(1)$ of finite index and $\bar{\Gamma}$ the group of matrices such that $\Gamma \cong \bar{\Gamma} /\{ \pm I\}$, that is, we assume that $-I \in \Gamma$. A congruence subgroup $\Gamma$ of level $N$ is a subgroup of $\Gamma(1)$ which satisfies $\Gamma(1) \supset \Gamma \supset \Gamma(N)$.

A fundamental region (in the loose sense) for $\Gamma(1)$ is the triangle $R=R(1)$ $=\{z=x+i y:|z|>1,|x|<1 / 2, y>0\}$ in the upper half plane. If $[\Gamma(1): \Gamma]=\mu$ and $\Gamma(1)=\sum_{k=1}^{\mu} V_{k} \Gamma$, then $R(\Gamma)=\bigcup_{k=1}^{\mu} V_{k} R$ is a fundamental region for $\Gamma$. The cusps of this fundamental region are the images of $\infty$. It is known that a coset decomposition can be effected in the form

$$
\bar{\Gamma}(1)=\bigcup_{j=1}^{\sigma} \bigcup_{k \bmod \lambda_{j}} U^{k} A_{j} \bar{\Gamma}
$$

where $\sigma$ is the number of cusps (inequivalent with respect to $\Gamma$ ) of a fundamental region; these are $\left\{A_{j}^{-1} \infty_{\infty}: j=1,2, \ldots, \sigma\right\}$. We assume that $A_{1}=I$. The number $\lambda_{j}$ is called the "width" of the fundamental region at $A_{j}^{-1} \infty$ and is the smallest positive integer such that $P_{j}=A_{j}^{-1} U^{\lambda_{j}} A_{j} \in \bar{\Gamma}$. We 
write simply $\lambda_{1}=\lambda$. If we set $p_{j}=A_{j}^{-1} \infty$ then $P_{j}$ is the parabolic transformation fixing $p_{j}$.

A character $v$ on $\bar{\Gamma}$ is a mapping from the group into the complex numbers of absolute value 1 for which

$$
v\left(V_{1} V_{2}\right)=v\left(V_{1}\right) v\left(V_{2}\right) \text {. }
$$

Throughout this paper we assume that

$$
v(-I)=1 \text {. }
$$

These characters (on $\bar{\Gamma}$ ) form a group isomorphic to the character group of $\Gamma$. A meromorphic function $F$ in $\mathscr{W}$ is called a modular form of dimension -2 for $\Gamma$ and the character $v$ if, for each $V=(a b \mid c d) \in \bar{\Gamma}$,

$$
F(V z)=v(V)(c z+d)^{2} F(z) .
$$

We further assume that $F$ has at most a finite number of poles (measured in the appropriate local variable) in any fundamental region for $\Gamma$. We say that $F$ belongs to the class $\Gamma,-2, v$ written $F \in\{\Gamma,-2, v\}$.

We shall need some further facts on characters. Let $v$ be a character on $\bar{\Gamma}$ and $V \in \bar{\Gamma}(1)$; then we say $v$ induces the character $v^{\prime}$ on $V \bar{\Gamma} V^{-1}$ when

$$
v^{\prime}\left(M^{\prime}\right)=v(M) \text { for } M^{\prime}=V M V^{-1} \in V \Gamma V^{-1} .
$$

We shall be particularly interested in the case $V=A_{j}$ and we simplify the notation to

$$
\Gamma_{j}=A_{j} \Gamma A_{j}^{-1}, \quad v_{j}\left(M^{\prime}\right)=v(M) .
$$

We set $\lambda_{j}=\lambda\left(A_{j}, \Gamma\right)$, the width at $A_{j}^{-1} \infty=p_{j}$, then

$$
\lambda_{j}=\lambda\left(A_{j}, \Gamma\right)=\lambda\left(I, \Gamma_{j}\right) .
$$

We also introduce $\kappa_{j}=\kappa\left(A_{j}, \Gamma\right)$ where $\kappa_{j}$ is defined by

$$
v\left(P_{j}\right)=e\left(\kappa_{j}\right), \quad 0 \leqq \kappa_{j}<1,
$$

for $P_{j}=A_{j}^{-1} U^{\lambda_{j}} A_{j}$. We have used the notation

$$
e(z)=e^{2 \pi i z} \text {. }
$$

We find that

$$
\kappa_{j}=\kappa\left(A_{j}, \Gamma\right)=\kappa\left(I, \Gamma_{j}\right) .
$$

To simplify the notation we write $\kappa=\kappa_{1}=\kappa(I, \Gamma)$.

If $F \in\{\Gamma,-2, v\}$ then at the cusp $p_{j}=A_{j}^{-1} \infty$ it has the expansion

$$
F(z)=\left(c_{j} z+d_{j}\right)^{-2} \sum_{n=s_{j}}^{\infty} a_{n}\left(F, A_{j}, \Gamma\right) e\left(\left(n+\kappa_{j}\right) A_{j} z / \lambda_{j}\right),
$$

where $s_{j}$ is a finite integer and $s_{j}+\kappa_{j}$ is the order of $F$ at $p_{j}$. The numbers $a_{n}\left(F, A_{j}, \Gamma\right)$ are called the "Fourier coefficients" in the expansion at $p_{j}$ 
$=A_{j}^{-1} \infty$ where $A_{j}=\left(a_{j} b_{j} \mid c_{j} d_{j}\right)$. In case $s_{j}+\kappa_{j} \geqq 0$ for $j=1,2, \cdots, \sigma$ and $F$ has no poles in $\mathscr{H}$, it is called an everywhere regular form. The everywhere regular forms in $\{\Gamma,-2, v\}$ form a complex vector space denoted by $\mathscr{C}^{+}(\Gamma,-2, v)$. A form in $\mathscr{C}^{+}$is called a cusp form provided $s_{j}+\kappa_{j}>0$ for $j=1,2, \cdots, \sigma$. The cusp forms form a subspace of $\mathscr{C}^{+}$denoted by $\mathscr{L}^{0}(\Gamma,-2, v)$. Both of these spaces are finite-dimensional. In dealing with expansions at cusps we find it convenient to introduce the $V$-transform of $F, F_{V}$, defined by

$$
F_{V}(z)=F(z) \mid V^{-1}=(-c z+a)^{-2} F\left(V^{-1} z\right)
$$

where $V=(a b \mid c d)$. If $F \in\{\Gamma,-2, v\}\left(\mathscr{C}^{+}, \mathscr{C}^{9}\right)$ then $F_{V} \in\left\{V \Gamma V^{-1},-2, v^{\prime}\right\}$ $\left(\mathscr{C}^{+}, \mathscr{C}^{9}\right)$ where $v^{\prime}$ is the character induced by $v$.

We define the following sets of integers

$$
\begin{aligned}
\mathscr{C}\left(A_{j}\right) & =\mathscr{C}\left(A_{j}, \Gamma\right)=\left\{c: \exists V=(\cdot \cdot \mid c \cdot) \in A_{j} \Gamma\right\}, \\
\mathscr{D}\left(c, A_{j}\right) & =\mathscr{D}\left(c, A_{j}, \Gamma\right)=\left\{d: \exists V=(\cdot \cdot c d) \in A_{j} \Gamma\right\}, \\
\mathscr{D}_{c}\left(A_{j}\right) & =\mathscr{D}_{c}\left(A_{j}, \Gamma\right)=\left\{d \in D\left(c, A_{j}\right): d \in[0, c \lambda]\right\}, \\
\mathscr{X}_{c}\left(A_{j}\right) & =\mathscr{A}_{c}\left(A_{j}, \Gamma\right)=\left\{a: \exists V=(a \cdot \mid c \cdot) \in A_{j} \Gamma, a \in\left[0, c \lambda_{j}\right]\right\},
\end{aligned}
$$

where $[0, c \lambda]$ means the closed interval of real numbers between 0 and $c \lambda$. One sees that

$$
\mathscr{D}\left(c, A_{j}\right)=\bigcup_{q=-\infty}^{\infty}\left\{d+c q \lambda: d \in \mathscr{D}_{c}\left(A_{j}\right)\right\} .
$$

We shall come across certain exponential sums, called Kloosterman sums, defined by

$$
\begin{aligned}
W_{c}(n, \mu) & =W_{c}\left(n+\kappa, A_{j}, \Gamma, \mu+\kappa_{j}\right) \\
& =\sum_{d \in \mathscr{\mathscr { V }}_{c}\left(A_{j}\right)} \bar{v}\left(A_{j}^{-1} V_{c, d}\right) e\left(\frac{n+\kappa}{c \lambda} d+\frac{\mu+\kappa_{j}}{c \lambda_{j}} a\right)
\end{aligned}
$$

where $V_{c, d}=(a b \mid c d) \in A_{j} \Gamma$. When $\Gamma=\Gamma(1), A_{j}=I$, and $v=1$ the sum in (2.15) becomes the original sum investigated by Kloosterman. In the following we shall assume that

$$
W_{c}(n, \mu)=O\left(|c|^{\xi}\right), \quad 1 / 2<\xi<1,
$$

for fixed $\mu$. In $\$ 6$ we discuss cases for which the estimate (2.16) is valid.

3. We consider the double series

$$
\sum_{c, d} \bar{v}\left(A_{j} V_{c, d}\right)(c z+d)^{-2} e\left(\left(\mu+{ }_{\kappa_{j}}\right) V_{c, d} z / \lambda_{j}\right)
$$

where the summation conditions are $c \in \mathscr{C}\left(A_{j}\right), d \in \mathscr{D}\left(c, A_{j}\right)$ and $V_{c, d}$ $=(a b \mid c d) \in A_{j} \Gamma$. We assume $\mu$ to be a rational integer for which $\mu+\kappa_{j}$ $\neq 0$. This double series is not absolutely convergent; hence, we shall have 
to define in what order the summation is to be carried out.

First, however, we remark that the terms of (3.1) are uniquely determined by $c$ and $d$ even though $V_{c, d}$ is not. Suppose $V_{c, d}^{\prime} \in A_{j} \Gamma$ then $V_{c, d}=U^{k_{\lambda j}} V_{c, d}^{\prime}$ for some integer $k$. Replacing $V_{c, d}$ by $U^{k_{j} j} V_{c, d}^{\prime}$ in the term of (3.1) containing $V_{c, d}$ and using the results $U^{a} z=z+a$ and

$$
v\left(A_{j}^{-1} U^{k_{j}} V_{c, d}^{\prime}\right)=v\left(A_{j}^{-1} U^{k_{j}} A_{j}\right) v\left(A_{j}^{-1} V_{c, d}^{\prime}\right)=e\left(k_{\kappa_{j}}\right) v\left(A_{j}^{-1} V_{c, d}^{\prime}\right),
$$

we obtain the result that the term determined by $V_{c, d}$ is equal to the term determined by $V_{c, d}^{\prime}$. We shall drop the subscripts $c$ and $d$ on $V$ whenever it is clear what is meant. In order to define the order of summation in (3.1) we introduce for $c \neq 0, c \in \mathscr{C}\left(A_{j}\right)$ the auxiliary series

$$
H(c, z)=\sum_{d=-\infty ; d \in \in(c, A)}^{\infty} v\left(A^{-1} V\right)(c z+d)^{-2} e((\mu+\kappa) V z / \lambda),
$$

where we have dropped the subscript $j$ on $A$ for convenience of notation and $\kappa=\kappa(A, \Gamma), \lambda=\lambda(A, \Gamma)$. We shall show that this series is absolutely uniformly convergent on compact subsets of $\mathscr{Z}$; that is, the series of absolute values converges uniformly on compact subsets of $\mathscr{Z}$. The double series (3.1) is to be summed thus:

$$
\delta(A, I) e((\mu+\kappa) z / \lambda)+\lim _{K \rightarrow \infty} \sum_{c=-K ; c \in \mathscr{L}(A)}^{-1} H(c, z)+\lim _{K \rightarrow \infty} \sum_{c=1 ; c \in \mathscr{L}(A)}^{K} H(c, z),
$$

where $\delta(A, I)=2$ if $A=I$ and 0 otherwise. If both limits in (3.3) exist simultaneously, we shall define (3.3) to be $G(z, v, A, \Gamma, \mu)$ and call it a Poincare series. We drop some of the parameters when no confusion can be caused. When $A=I$ the terms of (3.1) corresponding to $c=0$ occur for $d= \pm 1$. We can choose $V_{0, \pm 1}= \pm I$. This accounts for the first term of (3.3).

The following estimate for $|c z+d|$ is essential. For $z \in \mathscr{H}$,

$$
|c z+d| \geqq|d| \sin \delta, \quad 0<\arg z=\delta<\pi .
$$

For a proof consider the parallelogram with vertices $\pm|d|$ and $\pm|c| z$. Then $|c z+d|$ is the length of one side of this parallelogram, whereas $|d| \sin \delta$ is the length of the perpendicular segment from $|d|$ to the diagonal through $|c| z$ and $-|c| z$. Thus $|d| \sin \delta$ is the length of a leg of the right triangle whose hypotenuse is $|c z+d|$. This completes the proof. Next we note that

$$
|c z+d| \geqq|c| y \quad(z=x+i y, y>0) .
$$

We split $H(c, z)$ into the sum of two series using the relation $V z$ $=(a z+b) /(c z+d)=a / c-1 / c(c z+d)$ valid for $c \neq 0$. Define

$$
H_{1}(c, z)=\sum_{d=-\infty ; d \in \mathscr{C}(c, A)}^{\infty} \frac{e((\mu+\kappa) a / c \lambda)\{e(-(\mu+\kappa) / \lambda c(c z+d))-1\}}{v\left(A^{-1} V\right)(c z+d)^{2}}
$$


and

$$
H_{2}(c, z)=\sum_{d=-\infty ; d \in \mathcal{U}_{(c, A)}^{\infty}} \bar{v}\left(A^{-1} V\right)(c z+d)^{-2} e((\mu+\kappa) a / c \lambda)
$$

for $c \neq 0, c \in \mathscr{C}(A)$. Formally, $H(c, z)=H_{1}(c, z)+H_{2}(c, z)$. We show that $H_{1}$ and $H_{2}$ converge absolutely uniformly on compact subsets of $\mathscr{E}$. Expand the second exponential in (3.6) to obtain

$$
H_{1}(c, z)=\sum_{d=-\infty ; d \in U(c, A)}^{\infty} \sum_{m=1}^{\infty} \frac{e((\mu+\kappa) a / c \lambda)(-2 \pi i)^{m}(\mu+\kappa)^{m}}{v\left(A^{-1} V\right)(\lambda c)^{m} m !(c z+d)^{m+2}} .
$$

This double series is dominated by

$$
\sum_{d} \sum_{m=1}^{\infty} \frac{(2 \pi)^{m}(|\mu|+1)^{m}}{|c|^{m} \lambda^{m} m !|c z+d|^{m+2}}
$$

Using the estimates (3.4) and (3.5) we obtain for our dominating series the upper estimate

$$
\begin{aligned}
(c y)^{-2} \sum_{m=0}^{\infty} \frac{1}{m !}\left\{\frac{2 \pi(|\mu|+1)}{y}\right\}^{m} & \\
& +\left\{\frac{2}{|c|^{5 / 2} \sin \delta} \sum_{0}^{\infty} \frac{1}{m !}\left\{\frac{2 \pi(|\mu|+1)}{\sqrt{ }(y \sin \delta)}\right\}^{m}\right\}\left\{\sum_{1}^{\infty} \frac{1}{d^{3 / 2}}\right\} .
\end{aligned}
$$

therefore,

$$
\begin{aligned}
& \left|H_{1}(c, z)\right| \leqq(c y)^{-2} \exp [2 \pi(|\mu|+1) / y] \\
& +C|c|^{-5 / 2}(y \sin \delta)^{-1} \exp [2 \pi(|\mu|+1) / \sqrt{ }(y \sin \delta)],
\end{aligned}
$$

where $C$ is a constant independent of $z$. This implies that the series for $H_{1}(c, z)$ converges absolutely uniformly on the strip $|x| \leqq x_{0}, y \geqq y_{0}>0$.

Note that the series for $H_{2}(c, z)$ corresponds to the missing term $m=0$ in (3.8). The dominating series for $H_{2}(c, z)$ is $\sum_{d}|c z+d|^{-2}$. We obtain from (3.4) and (3.5) the result

$$
\left|H_{2}(c, z)\right| \leqq(c y)^{-2}+\pi^{2} / 3 \sin ^{2} \delta
$$

which shows that the series for $H_{2}(c, z)$ converges absolutely uniformly on the strip $|x| \leqq x_{0}, y \geqq y_{0}>0$. This shows that $H(c, z)=H_{1}(c, z)$ $+H_{2}(c, z)$ is regular in $\mathscr{W}$. We shall want to sum these functions on $c$ and the estimate (3.10) is not good enough for this purpose.

The terms of $H_{2}(c, z)$ in (3.7) are uniquely determined by the choice of $c$ and $d$. We make the choice of $a$ in $V$ unique by requiring that $a \in \mathscr{S}_{c}(A)$ (this can be justified by considering $U^{k \lambda_{j}} V_{c, d}$ ). Now dividing $d$ by $c \lambda$ we obtain $d=q c \lambda+d^{\prime}$ with $d^{\prime} \in \mathcal{Y}_{c}(A)$. Thus $V_{c, d}=V_{c, d^{\prime}} U^{q}$

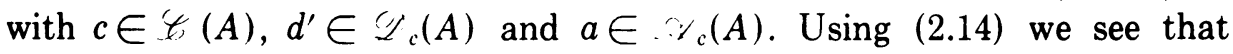




$$
H_{2}(c, z)=\sum_{d \in \mathbb{U}_{c}(A)} \frac{e\left(\left(\mu+\kappa_{j}\right) a / c \lambda_{j}\right)}{v\left(A_{j}^{-1} V\right)} \sum_{q=-\infty}^{\infty} \frac{e\left(-q_{\kappa}\right)}{(c z+d+q c \lambda)^{2}},
$$

where we have used the relation $1 / v\left(U^{q \lambda}\right)=e\left(-q_{\kappa}\right)$ and reintroduced the subscript $j$ on $A, \kappa$, and $\lambda$ since $\kappa=\kappa_{1}$ and $\lambda=\lambda_{1}$ also appear in this form of $H_{2}(c, z)$. We apply the Lipschitz formula [1, p. 206] to the inner sum (on $q$ ) to obtain

$$
(-2 \pi i / c \lambda)^{2} \sum_{n=0}^{\infty}(n+\kappa) e((n+\kappa)(z / \lambda+d / c \lambda))
$$

hence

$$
H_{2}(c, z)=(-2 \pi i / c \lambda)^{2} \sum_{n=0}^{\infty}(n+\kappa) W_{c}(n, \mu) e((n+\kappa) z / \lambda),
$$

where we have interchanged the summations and introduced the Kloosterman sum (2.15). Using the estimate (2.16) for the Kloosterman sum and the fact that $0 \leqq \kappa<1$, we find that

$$
\begin{aligned}
\left|H_{2}(c, z)\right| & \leqq \frac{C(\xi)}{|c|^{2-\xi}} \sum_{n=0}^{\infty}(n+1) \exp (-2 \pi n y / \lambda) \\
& =C(\xi)|c|^{\xi-2}\left(1-e^{-2 \pi y / \lambda}\right)^{-2}
\end{aligned}
$$

(where $-3 / 2<\xi-2<-1$ ). It is now easy to see using the estimates (3.9) and (3.12) that the series $\sum_{c} H_{1}(c, z)$ and $\sum_{c} H_{2}(c, z)$ converge absolutely uniformly for $|x| \leqq x_{0}, y \geqq y_{0}>0$, where $c$ is summed over $\mathscr{C}(A)$, $c \neq 0$. This shows that if the limits in (3.3) exist (and they do if the Kloosterman sum has the estimate (2.16)) then the function so defined is regular in $\mathscr{E}$. This completes the proof of the following lemma.

Lemma 1. Assuming the estimate (2.16) for the Kloosterman sum $W_{c}(n, \mu)$, the functions $G\left(z, v, A_{j}, \Gamma, \mu\right)$ defined by (3.3) are regular in $\mathscr{H}$. Furthermore, we have the expressions

$$
G\left(z, v, A_{j}, \Gamma, \mu\right)=\delta\left(A_{j}, I\right) e((\mu+\kappa) z / \lambda)+\sum_{c \in t\left(A_{j}\right) ; c \neq 0} H(c, z)
$$

and

$$
\begin{aligned}
& G\left(z, v, A_{j}, \Gamma, \mu\right)=\delta\left(A_{j}, I\right) e((\mu+\kappa) z / \lambda) \\
& +\sum_{c \in\left(A_{j}\right) ; c \neq 0} H_{1}(c, z)+\sum_{c \in !\left(A_{j}\right) ; c \neq 0} H_{2}(c, z),
\end{aligned}
$$

where the three series are absolutely convergent on compact subsets of $\mathbb{E}$. The functions $H(c, z), H_{1}(c, z)$ and $H_{2}(c, z)$ are defined in (3.2), (3.6) and (3.7), respectively. 
We conclude this section with some estimates which will be useful later. r. $\mathrm{t}$

$$
H_{1}(z)=\sum_{c \in \mathcal{Y},(A)}^{\prime} \sum_{d \in \in(\mathcal{N}, A)} \frac{e((\mu+\kappa) a / c \lambda)\{e(-(\mu+\kappa) / c \lambda(c z+d))-1\}}{v\left(A^{-1} V\right)(c z+d)^{2}} .
$$

This double series is absolutely and uniformly convergent in the strip $|x| \leqq x_{0}, y \geqq y_{0}>0$ as we have seen. We find that

$$
\begin{aligned}
&\left|H_{1}(z)\right| \leqq C y^{-2} \exp [2 \mu(|\mu|+1) / y] \\
&+C(y \sin \delta)^{-1} \exp [2 \pi(|\mu|+1) / \sqrt{ }(y \sin \delta)]
\end{aligned}
$$

making use of the estimate (3.9). If we let $H_{2}(z)=\sum_{c} H_{2}(c, z)$, where summation is extended over $\mathscr{C}(A), c \neq 0$, we find that

$$
\left|H_{2}(z)\right| \leqq C(\xi, \Gamma)\left(1-e^{-2 x y / \lambda}\right)^{-2} .
$$

Then.(3.14) takes the more compact form

$$
G\left(z, v, A_{j}, \Gamma, \mu\right)=\delta\left(A_{j}, I\right) e((\mu+\kappa) z / \lambda)+H_{1}(z)+H_{2}(z) .
$$

4. As we have mentioned the main tool of this paper is the Rademacher Lemma. In this section we prove this lemma in the generality which we need. Before stating the lemma we introduce some notation.

By a lattice point for $A \bar{\Gamma}$ we mean an ordered pair of integers $(c, d)$ which come from the lower row of a matrix $V=(a b \mid c d) \in A \bar{\Gamma}$ (we of course assume that $A \in \bar{\Gamma}(1))$. Let $\mathscr{L}(A)=\mathscr{L}(A, \bar{\Gamma})$ be the set of lattice points for $A \bar{\Gamma}$ :

$$
\mathscr{L}(A)=\{(c, d): V=(\cdot \cdot \mid c d) \in A \bar{\Gamma}\} .
$$

Let $\mathscr{Y}(K)$ denote a square in the $(u, v)$-plane of side $2 K$ centered at the origin with sides parallel to the $u$ and $v$ axes (that is, the set $\{(u, v) ;|u|,|v| \leqq K\})$. Let

$$
\mathscr{L}(K, A)=\mathscr{f}(K) \cap \mathscr{f}(A)
$$

be the lattice points for $A \bar{\Gamma}$ which lie in $\mathcal{f}(K)$. If $M=(\alpha \beta \mid \gamma \delta) \in \bar{\Gamma}(1)$ we let $M$ act as a homogeneous transformation on the plane containing the lattice points given by

$$
(u, v) M=\left(u^{\prime}, v^{\prime}\right)=(\alpha u+\gamma v, \beta u=\delta v) .
$$

We note that if $(c, d) \in \mathscr{f}(A)$ and $M \in \bar{\Gamma}$, then $\left(c^{\prime}, d^{\prime}\right) \in \mathscr{L}(A)$. For $M \in \bar{\Gamma}$ (4.3) defines a mapping that is one-to-one from $\mathscr{L}(A, \bar{\Gamma})$ onto $\mathscr{L}(A, \bar{\Gamma})$. Next, we note that the image of $\mathscr{f}(K), \mathscr{f}(K) M$, is a parallelogram centered at the origin. The lattice points in $\mathscr{S}(K) M$ are

$$
\mathscr{L}(K, A) M=\{(c, d) \in \mathscr{L}(A):(c, d) \in \mathscr{f}(K) M\} .
$$


Let $v=g(u)$ be the "upper boundary curve" of the parallelogram $\mathscr{S}(1) M$, thus a polygonal line. Let

$$
u_{1}=\max \{u:(u, v) \in \mathscr{Y}(1) M\} \text {. }
$$

Clearly $u_{1}>0$. We see that either $g(u)>0$ for $\left[-u_{1}, u_{1}\right]$, for example, when $\mathscr{f}(1) M$ is a square, or $g(u)$ has a zero in one or the other of the intervals $\left[-u_{1}, 0\right),\left(0, u_{1}\right]$ (but not both). Further, note that if $g\left(u_{1}\right)=0$ then $g\left(-u_{1}\right)=0$. We let $u_{0}$ denote the zero of $v=g(u)$ if it has one, otherwise let $u_{0}=u_{1}$. Then in either case

$$
g\left(u_{0} / 2\right)>0 .
$$

Finally, the upper boundary curve of $\mathscr{S}(K) M$ is $v=K g(u / K)$, and the lower boundary curve is $v=-K g(-u / K)$. Now we are in a position to state our lemma.

LEMMA 2. If the Kloosterman sums (2.15) have the estimate (2.16) then for $M \in \bar{\Gamma}(1)$

$$
\begin{array}{r}
\lim _{K \rightarrow \infty} \sum_{c=-K ; c \in \mathscr{C}(A) ; c \neq 0}^{K} \sum_{d=-\infty ; d \in \mathscr{D}(c, A)}^{\infty} \bar{v}\left(A^{-1} V\right)(c z+d)^{-2} e((\mu+\kappa) a / c \lambda) \\
=\lim _{K \rightarrow \infty} \sum_{(c, d) \in \mathscr{L}(K, A) M ; c \neq 0} \bar{v}\left(A^{-1} V\right)(c z+d)^{-2} e((\mu+\kappa) a / c \lambda),
\end{array}
$$

where $V=(a b \mid c d) \in A \bar{\Gamma}$ and $\mathscr{L}(K, A) M$ is defined in (4.4).

This lemma roughly states that the double series in the left member of (4.5) can be summed over expanding parallelograms. We shall try and simplify the notation by writing

$$
\sum_{c=-L}^{K}(A) \sum_{d=-\infty}^{\infty}(A) \text { in place of } \sum_{c=-L ; c \in \mathscr{L}(A)}^{K} \sum_{d=-\infty ; d \in \mathscr{L}(c, A)}^{\infty} .
$$

If a prime appears on a summation sign, then the value $c=0$ is omitted. Equation (4.5) becomes

$$
\text { (4.6) } \sum_{c=-\infty}^{\infty}(A) H_{2}(c, z)=\lim _{K \rightarrow \infty} \sum_{(c, d) \in \mathcal{L}(K, A) M}^{\prime} \bar{v}\left(A^{-1} V\right) e((\mu+\kappa) a / c \lambda)(c z+d)^{-2} \text {, }
$$

where $H_{2}(c, z)$ is defined in (3.7). We have shown that the series on the left of (4.6) is absolutely uniformly convergent for $y \geqq y_{0}>0,|x| \leqq x_{0}$. On account of this it suffices to show that

$$
\sum_{c=-u_{1} K}^{u_{1} K}(A)\left\{\sum_{d>K g(c / K)}(A) \cdots+\sum_{d<-K g(-c / K)}(A) \cdots\right\}
$$

can be made arbitrarily small. These two double sums are equal since if $(c, d)$ gives rise to a term in one then, $(-c,-d)$ give rise to a term in the 
other and the summands are the same. Thus, it suffices to show that the first drible series in (4.7) can be made small by taking $K$ large.

Following Lehner [10] let

$$
g(c, d)=\left\{\begin{array}{l}
\bar{v}\left(A_{j}^{-1} V\right) e\left(\left(\mu+\kappa_{j}\right) a / c \lambda_{j}\right) \text { for }(c, d) \in \mathscr{L}\left(A_{j}\right), \\
0 \text { for } c, d \text { rational integers, }(c, d) \notin \mathscr{L}\left(A_{j}\right) .
\end{array}\right.
$$

We set $\phi(c, d)=e\left(d_{\kappa} / c \lambda\right) g(c, d)$. Let $V=(a b \mid c d) \in A_{j} \bar{\Gamma}$ then

$$
V U^{\lambda}=(a \cdot \mid c d+c \lambda) \in A_{j} \bar{\Gamma} \text { and } g(c, d+c \lambda)=\bar{v}\left(A_{j}^{-1} V U\right) e\left(\left(\mu+\kappa_{j}\right) a / c \lambda_{j}\right) \text {. }
$$

Thus $\phi(c, d+c \lambda)=e\left(d_{\kappa} / c \lambda\right) e(\kappa) \bar{v}\left(A_{j}^{-1} V\right) e(-\kappa) e\left(\left(\mu+\kappa_{j}\right) a / c \lambda_{j}\right)=\phi(c, d)$. The function $\phi(c, d)$ is a periodic function on the integers with the period $c \lambda$ so that it has the finite Fourier expansion:

$$
\phi(c, d)=\sum_{k \bmod |c| \lambda} B_{k} e(k d / c \lambda), \quad B_{k}=\frac{1}{|c| \lambda} \sum_{d \bmod c} \phi(c, d) e(-k d / c) .
$$

This in turn implies that

$$
g(c, d)=\sum_{k \bmod |c| \lambda} B_{k} e((k-\kappa) d / c \lambda)
$$

and

$$
B_{k}=\frac{1}{|c| \lambda} \sum_{d \bmod |c| \lambda} \bar{v}\left(A_{j}^{-1} V\right) e\left(\left(\mu+\kappa_{j}\right) a / c \lambda_{j}+(-k+\kappa) d / c \lambda\right) .
$$

We see from the definition (2.15) of the Kloosterman sum that

$$
B_{k}=(1 /|c| \lambda) W_{c}\left(-k+\kappa, A_{j}, \mu+\kappa_{j}\right) .
$$

The introduction of $g(c, d)$ allows us to drop the complicated conditions on the summation variables. We write the first sum in (4.7) in the following way:

$$
W(K)=\sum_{c=-u_{1} K}^{u_{1} K} \sum_{d>K g(c / K)} \frac{g(c, d)}{(c z+d)^{2}} .
$$

In order to estimate this sum, we consider three cases which depend upon the shape of the upper boundary curve, $v=g(u)$, of $S(1) M$. We have seen that either $g(u)>0$ for $-u_{1} \leqq u \leqq u_{1}$ or $g\left(u_{0}\right)=0, u_{0} \neq 0,-u_{1}<u_{0}<u_{1}$, or $g\left(u_{1}\right)=0$ (in which case $g\left(-u_{1}\right)=0$ ). We break the double sum (4.12) into one, two or three double sums in order to take advantage of the fact that either the $c$ 's or the $d$ 's are bounded away from 0 be a positive multiple of $K$. We describe this more fully. Suppose $g(u)>0$ for $-u_{1} \leqq u$ $\leqq u_{1}$, then we shall be able to estimate $W(K)$ as it stands since $d>K g(c / K)$ $>K \beta$ with $\beta>0$. Next, suppose $g\left(u_{0}\right)=0$ with $-u_{1}<u_{0}<0$ (the case $0<u_{0}<u_{1}$ would be handled in an obviously similar way). Here we would consider 


$$
W(K)=\sum_{c=-u_{1} K}^{u_{0} K / 2} \sum_{d>K_{g}(c / K)} \cdots+\sum_{c=-u_{1} K / 2}^{u_{1} K / 2} \sum_{d>K g(c / K)} \cdots
$$

Finally, if $g\left(u_{1}\right)=0$ then we write

$$
W(K)=\sum_{u_{1} K>|c|>u_{1} K / 2} \sum_{d>K_{g}(c / K)} \cdots+\sum_{c=-u_{1} K / 2}^{u_{1} K / 2} \sum_{d>K_{g}(c / K)} \cdots
$$

The proof of the lemma has been reduced to showing that $W(K)$ defined in (4.12) is $o(1)$. This will be accomplished if we can show that the sums in (4.13) or (4.14) or $W(K)$ itself, depending upon the location of the zero of $v=g(u)$, can be estimated as $o(1)$. We, therefore consider double series of the following types: first,

$$
W_{1}(K)=\sum_{c=\alpha K}^{\beta K} \sum_{k=0}^{|c| \lambda-1} B_{k} \sum_{d>K g(c / K)}(c z+d)^{-2} e((k-\kappa) d / c \lambda)
$$

with fixed $\alpha$ and $\beta$,

$$
0<\alpha<\beta \text { and }|K g(c / K)|<\infty \quad \text { in } \alpha K \leqq c \leqq \beta K ;
$$

and, second,

$$
W_{2}(K)=\sum_{c=\alpha K}^{c=\beta K} \sum_{k=0}^{|c| \mid \lambda-1} B_{k} \sum_{d>K_{g}(c / K)} \frac{e\left((k-\kappa)^{d} / c \lambda\right)}{(c z+d)^{2}},
$$

where

$$
\alpha<0<\beta \text { and } g(c / K)>0 \text { for } \alpha K<c<\beta K .
$$

In the equations (4.15) and (4.16) we have introduced the Fourier expansion for $g(c, d)$ as given by (4.9) with $B_{k}$ defined in (4.11). As we have already mentioned the estimation of $W(K)$ reduces to showing that sums of the type (4.15) and (4.16) have the estimate $o(1)$.

We estimate the inner sums in (4.15) and (4.16) using the method of partial summation $\left({ }^{2}\right)$. Let

$$
S_{d}=\sum_{t=0}^{d} e(\sigma(k-\kappa) t / c \lambda)
$$

with $\sigma= \pm 1$. Then $S_{0}=1$ and for $d \geqq 1$

$$
S_{d}=[1-e(\sigma(k-\kappa)(d+1) / c \lambda)] /[1-e(\sigma(k-\kappa) / c \lambda]
$$

if $k-\kappa \neq 0$. From the inequality $\sin \pi x \geqq \min \{2 x, 2-2 x\}$ for $0<x<1$, we find that $\left|S_{d}\right|<(|c| \lambda / 2)\left(|k-\kappa|^{-1}+[|c| \lambda-|k-\kappa|]^{-1}\right)=A(k)|c| \lambda / 2$. Under the conditions on $k$ and $\kappa$ the right member of (4.18) is larger than

(2) We are indebted to Professor Rademacher for the suggestion to use partial summation in the estimation process. 
1 ; therefore, this inequality also holds for $d=0$.

" recall the estimates (3.4) and (3.5) for $|c z+d|$. Let $\omega=\omega(z)$ $=\min \{\sin \delta, y\}$ where $0<\delta=\arg z<\pi$. Then for $0 \leqq \eta \leqq 1$

$$
|c z+d| \geqq \omega|c|^{1-\eta}|d|^{\eta}
$$

Let

$$
T(k, c, K)=\sum_{d>K g(c / K)} e((k-\kappa) d / c \lambda)(c z+d)^{-2}
$$

be the inner sum in (4.16). We estimate (4.20), first, with $k=0$. We have

$$
|T(0, c, K)| \leqq \sum_{d>K g(c / K)}|c z+d|^{-2}<\omega^{-2}|c|^{1-\eta} \sum_{d>K g(c / K)}|d|^{-1-\eta}
$$

If we let $Q=\min g(c / K)$ for $\alpha K \leqq c \leqq \beta K$ then under the conditions in (4.16a), $Q>0$ and does not depend upon $K$. Using an integral to give an upper estimate for the series in (4.21), we obtain

$$
|T(0, c, K)| \leqq C|c|^{-1+\eta} K^{-\eta},
$$

where $C$ is a general positive constant. Now we estimate $T(k, c, K)$ when $1 \leqq k \leqq|c| \lambda-1$. Here $k-\kappa \neq 0$. Introducing $S_{d}$ into the series we find

$$
T(k, c, K)=\sum_{d>K g(c / K)} S_{d}\left[(c z+d)^{-2}-(c z+d+1)^{-2}\right]-S_{K m}(c z+K m+1)^{-2}
$$

with $m=g(c / k)$. Thus

$$
\begin{aligned}
|T(k, c, K)| \leqq & \sum_{d>K m}\left|S_{d}\right|\left[|c z+d|^{-2}|c z+d+1|^{-2}\right. \\
& \left.+2|c z+d|^{-1}|c z+d+1|^{-2}\right]+\left|S_{K m}\right||c z+K m+1|^{-2} .
\end{aligned}
$$

Now using the estimates (4.18), (4.19) and an integral estimate for the series $\sum_{d>K g(c / K)}|d|^{-1-\eta}$, we obtain

$$
|T(k, c, K)| \leqq C A(k)|c|^{-1+\eta} K^{-\eta}
$$

for some choice of $\eta$ to be made later. Now we can estimate $W_{2}(K)$. We have

$$
\begin{aligned}
\left|W_{2}(K)\right| & \leqq \sum_{c=\alpha K}^{\beta K}\left\{\left|B_{0}\right||T(0, c, K)|+\sum_{k=1}^{|c| \lambda-1}\left|B_{k}\right||T(k, c, K)|\right\} \\
& =\sum_{c=\alpha K}^{\beta K}\left\{C(\xi)|c|^{-2+\xi+\eta} K^{-\eta}+C(\xi)|c|^{-2+\xi+\eta} K^{-\eta} \sum_{k=1}^{|c| \lambda-1} A(k)\right\}
\end{aligned}
$$

introducing the estimates above for $T(k, c, K)$ and the estimate on $B_{k}$ determined by (4.11) and (2.16). With the aid of an integral we obtain

$$
\sum_{k=1}^{|c| \lambda-1} A(k)=O(\log |c|) \text {. }
$$

Thus, 


$$
\left|W_{2}(K)\right| \leqq C(\xi) K^{-\eta} \sum_{2}^{\infty} c^{-2+\xi+\eta} \log c .
$$

Recall that $0 \leqq \eta \leqq 1$ and $1 / 2<\xi<1$; we choose $\eta>0$ so that the series on the right converges. Then

$$
W_{2}(K)=O\left(K^{-\eta}\right),
$$

where the constant in the $O$-symbol depends upon $\xi, z, \eta$ but is independent of $K$.

Now we turn to the estimation of $W_{1}(K)$. Let

$$
U(k, c, K)=\sum_{d>K_{g}(c / K)} e((k-\kappa) d / c \lambda)(c z+d)^{-2},
$$

where the conditions in (4.15a) are satisfied. There are two possibilities as to the sign of $g(c / K)$. We shall suppose $g(c / K)<0$; the other case is slightly simpler and the same methods are used in carrying out the estimation. Let

$$
\begin{aligned}
U(k, c, K)= & U_{1}(k, c, K)+U_{2}(k, c, K) \\
= & \sum_{d=1}^{-K g(c / K)-1} e(-(k-\kappa) d / c \lambda)(c z-d)^{-2} \\
& +\sum_{d=1}^{\infty} e((k-\kappa) d / c \lambda)(c z+d)^{-2} .
\end{aligned}
$$

Again estimating first the case $k=0$ we find

$$
|U(0, c, K)| \leqq 2 \sum_{d=1}^{\infty}|c z+d|^{-2} \leqq C(\eta)|c|^{-1+\eta} .
$$

Now we assume that $1 \leqq k \leqq|c| \lambda-1$, in which case $k-\kappa \neq 0$. As before we introduce $S_{d}$ into the sum $U(k, c, K)$. Thus

$$
\begin{aligned}
U_{1}(k, c, K)= & \sum_{d=1}^{-K m-2}\left\{S_{d}(c z-d)^{-2}-(c z-d-1)^{-2}\right\} \\
& +S_{-m K-1}(c z+m K+1)^{-2}-(c z-1)^{-2}
\end{aligned}
$$

with $m=g(c / K)$. The estimates (4.18) and (4.19) imply that

$$
\begin{aligned}
& \left|U_{1}(k, c, K)\right| \leqq(|c| \lambda A(k) / 2) \\
& \quad\left\{\omega^{-4}|c|^{-2+\eta} \sum_{d=1}^{\infty}|d|^{-2-\eta}+\omega^{-3}|c|^{-2+\eta} \sum_{d=1}^{\infty}|d|^{-1-\eta}+2 \omega^{-2}|c|^{-2}\right\} .
\end{aligned}
$$


Hence, wo find that $\left|U_{1}(k, c, K)\right| \leqq C(\eta)|c|^{-1+\eta} A(k)$. In the same way we fad that $\left.\mid L^{\prime} k, c, K\right)\left.|<C(\eta)| c\right|^{-1+\eta} A(k)$. This then implies that

$$
|U(k, c, K)|<C(\eta)|c|^{-1+\eta} A(k)
$$

for $k=1,2, \cdots,|c| \lambda-1$. We, therefore, obtain from (4.15) and the estimates (4.26), (4.27) and (2.16) the following estimate

$$
\left|W_{1}(K)\right| \leqq C(\xi, \eta) \sum_{c=\alpha K}^{\beta K}|c|^{-2+\xi+\eta}(1+\log |c|),
$$

where we have also used (4.23.1). Since $1 / 2<\xi<1$ we can choose $\eta, \epsilon$ so that $-2+\xi+\eta+\epsilon<-1$; hence,

$$
W_{1}(K)=O\left(K^{-1+\xi+\eta+\iota}\right) \text {. }
$$

The constant depends upon $\xi, \eta, \epsilon$ and $z$ but not on $K$. This completes the proof of the Rademacher Lemma.

The following is a corollary to Lemma 1 and Lemma 2.

LEMмA 3. If the Kloosterman sums (2.15) have the estimate (2.16) then for $M \in \bar{\Gamma}(1)$

$$
G\left(z, v, A_{j}, \Gamma, \mu\right)=\lim _{K \rightarrow \infty} \sum_{(c, d) \in \mathcal{Y}\left(K, A_{j}\right) M} \bar{v}\left(A_{j}^{-1} V\right)(c z+d)^{-2} e\left(\left(\mu+\kappa_{j}\right) V z / \lambda_{j}\right) .
$$

where $\mathscr{L}\left(K, A_{j}\right) M$ is defined in (4.4).

Proof. In equation (3.18) the double series for $H_{1}(z),(3.15)$, is absolutely convergent; hence, it may be rearranged in any manner. In particular it is the limit of its partial sums arranged as above. The Rademacher Lemma states this is also true for $\mathrm{H}_{2}(z)$.

5. In this section we state and prove the main theorem now that the ground work has been laid.

THEOREM 1. Let $\Gamma$ be a subgroup of finite index in the modular group $\Gamma(1)$, and let $v$ be a character on $\Gamma$. Let $A_{j}^{-1} \infty=p_{j}, j=1,2, \cdots, \sigma$ be a complete set of inequivalent cusps for $\Gamma, A_{j} \in \Gamma(1)$ and $A_{1}=I$. Then the double series

$$
\begin{aligned}
& G\left(z, v, A_{j}, \Gamma, \mu\right) \\
&=\sum_{c=-\infty ; c \in \zeta(A, \Gamma)} \sum_{d=-\infty ; d \in \mathcal{C}^{\prime}(c, A, \Gamma)}^{\infty} \bar{v}\left(A_{j}^{-1} V\right)(z+d)^{-2} e\left(\left(\mu+\kappa_{j}\right) V z / \lambda_{j}\right)
\end{aligned}
$$

when summed in the order

$$
\lim _{K \rightarrow \infty} \sum_{c=-K ; c \in \in^{\prime},\left(A_{j}, \Gamma\right)}^{K} \sum_{d=-\infty ; d^{\prime} /\left(c, A_{j}, \Gamma\right)}^{\infty} \bar{v}\left(A_{j}^{-1} V\right)(c z+d)^{-2} e\left(\left(\mu+\kappa_{j}\right) V z / \lambda_{j}\right)
$$


is a modular form of dimension -2 for $\Gamma$ and the character $v$, provided that $\mu+\kappa_{j} \neq 0, \mu$ an integer and $\kappa_{j}$ defined in (2.8), and that the Kloosterman sums

$$
\begin{aligned}
& W_{c}\left(n+\kappa, A_{j} A_{k}, A_{k}^{-1} \Gamma A_{k}, \mu+\kappa_{j}\right) \\
& =\sum_{d \in \mathscr{U}_{c}\left(A_{j} A_{k}, A_{k}^{-1} A_{k}\right)} \bar{v}_{k}\left(\left(A_{j} A_{k}\right)^{-1} V\right) e\left(\frac{(n+\kappa) d}{c \lambda}+\frac{\left(\mu+\kappa_{j}\right)}{c \lambda_{j}} a\right)
\end{aligned}
$$

$(j, k=1,2, \cdots, \sigma)$ have the estimate $O\left(|c|^{\xi}\right)$ with $1 / 2<\xi<1$.

The character $v_{k}$ in (5.2) is the character on $A_{k}^{-1} \Gamma G_{k}$ induced by $v$ on $\Gamma$, and the matrix.$V$ in (5.1) and (5.2) has the lower row $(c, d)$. The sets $\mathscr{C}_{5}\left(A_{j}\right)$, Y: $\left(c, A_{j}\right)$ and $\mathscr{C}_{c}\left(A_{j}\right)$ are defined in (2.13). Moreover, we have the Fourier expansion

$$
\begin{aligned}
& G\left(z, v, A_{j}, \Gamma, \mu\right) \\
& \quad=\delta\left(A_{j}, I\right) e((\mu+\kappa) z / \lambda)+\sum_{n+\kappa>0} c_{n}\left(v, A_{j}, \Gamma, \mu\right) e((n+\kappa) z / \lambda),
\end{aligned}
$$

where

$$
\begin{aligned}
c_{n}= & -\frac{4 \pi}{\lambda}\left|\frac{\lambda_{j}}{\mu+\kappa_{j}} \cdot \frac{n+\kappa}{\lambda}\right|^{1 / 2} \\
& \sum_{c=1 ; c \in C\left(A_{j}\right)}^{\infty} \frac{W_{c}\left(n+\kappa, A_{j}, \mu+\kappa_{j}\right)}{c} J_{1}\left(\frac{4 \pi}{c} \sqrt{ }\left(\frac{\mu+\kappa_{j}}{\lambda_{j}} \cdot \frac{n+\kappa}{\lambda}\right)\right),
\end{aligned}
$$

$\delta(A, I)=2$ if $A=I, 0$ otherwise, and $J_{1}(z)$ is the Bessel function of order 1 of the first kind.

Proof. We have shown in Lemma 1 that the double series (5.1) when summed in the manner specified converges uniformly to a function regular in $\mathscr{K}$. We now show that for $M=(\alpha \beta \mid \gamma \delta) \in \bar{\Gamma}$ the functional equation

$$
G\left(M z, v, A_{j}, \Gamma, \mu\right)=v(M)(\gamma z+\delta)^{-2} G\left(z, v, A_{j}, \Gamma, \mu\right)
$$

is satisfied. By Lemma 3,

$$
\begin{gathered}
G\left(M z, v, A_{j}, \Gamma, \mu\right) \\
=\lim _{K \rightarrow \infty} \sum_{(c, d) \in ! /\left(x, A_{j}\right)} v\left(A_{j}^{-1} V\right)(c M z+d)^{-2} e\left(\left(\mu+\kappa_{j}\right) V M z / \lambda_{j}\right) \\
=v(M)(\gamma z+\delta)^{2} \lim _{K \rightarrow \infty} \sum_{(c, d) \in \mathcal{Y}\left(\alpha, A_{j}\right)} \bar{v}\left(A_{j}^{-1} V\right)((c \alpha+d \gamma) z+c \beta+d \delta)^{-2} \\
\quad \cdot e\left(\left(\mu+\kappa_{j}\right) V M z / \lambda_{j}\right) \\
=v(M)(\gamma z+\delta)^{2} \lim _{K \rightarrow \infty} \sum_{(c, d) \in \mathcal{Y}\left(x, A_{j}\right) M} \bar{v}\left(A_{j}^{-1} V\right)(c z+d)^{-2} e\left(\left(\mu+\kappa_{j}\right) V z / \lambda_{j}\right),
\end{gathered}
$$

where we have replaced the summation variables $(c, d)$ by $(c \alpha+d \gamma, c \beta+d \delta)$. 
By Lemma 3 the last limit in the above equation is nothing more than $\left.G(z), A_{j}, \Gamma, \mu\right)$. To complete the proof that $G\left(z, v, A_{j}, \Gamma, \mu\right)$ is a modular form of dimension -2 for $\Gamma$ and $v$, we must show that the regularity conditions are satisfied. We have shown $G\left(z, v, A_{j}, \Gamma, \mu\right)$ to be regular in $\mathscr{Z}$, thus we must only show that these functions have at most polar singularities at the cusps $p_{j}=A_{j}^{-1} \infty, j=1,2, \cdots, \sigma$. This will follow once we have the Fourier expansion (5.3) and (5.4).

We begin with

$$
\begin{aligned}
H_{2}(z) & =\sum_{c=-\infty}^{\infty}(A) H_{2}(c, z) \\
& =\sum_{c=-\infty}^{\infty}(A)\left(\frac{-2 \pi i}{c \lambda}\right)^{2} \sum_{n=0}^{\infty}(n+\kappa) W_{c}(n, \mu) e((n+\kappa) z / \lambda),
\end{aligned}
$$

using the results of (3.11). This series is absolutely convergent for $y \geqq y_{0}>0$ and $|x| \leqq x_{0}$. Interchanging orders of summation,

$$
H_{2}(z)=\sum_{n=0}^{\infty} e((n+\kappa) z / \lambda) \sum_{c=-\infty}^{\infty}\left(A_{j}\right)(n+\kappa)\left(\frac{-2 \pi i}{c \lambda}\right)^{2} W_{c}\left(n, A_{j}, \mu\right) .
$$

We have shown the double series (3.15) for $H_{1}(z)$ to be absolutely convergent. We derive for $H_{1}(z)$ a representation similar to (5.5) for $H_{2}(z)$. We start by applying the Lipschitz formula [l, p. 206] to the summation on $d$ in (3.8). We obtain upon interchanging orders of summation and introducing the Kloosterman sums

$$
\sum_{d=-\infty}^{\infty}\left(A_{j}\right) \frac{e\left(\left(\mu+\kappa_{j}\right) a / c \lambda_{j}\right)}{v\left(A_{j}^{-1} V\right)(c z+d)^{m+2}}=\frac{(-2 \pi i)^{2 m+2}}{\Gamma(m+2)} \sum_{n=0}^{\infty}(n+\kappa)^{m+1} \frac{W_{c}(n, \mu)}{(c \lambda)^{m+2}}
$$

Hence,

$$
\begin{aligned}
H_{1}(z)= & \sum_{n=0}^{\infty} e((n+\kappa) z / \lambda) \\
& \cdot \sum_{m=1}^{\infty} \sum_{c=-\infty}^{\infty}\left(A_{j}\right) \frac{(-2 \pi i)^{2 m+2}(n+\kappa)^{m+1}(\mu+\kappa)^{m} W_{c}(n, \mu)}{c^{2 m+2} \lambda_{j}^{m} \lambda^{m+2} m !(m+1) !}
\end{aligned}
$$

On comparing (5.5) and (5.6) we see that (5.5) is just the missing term $m=0$ in (5.6). From (3.18) we see that

$$
G\left(z, v, A_{j}, \Gamma, \mu\right)=\delta\left(A_{j}, I\right) e((\mu+\kappa) z / \lambda)+\sum_{n=0}^{\infty} c_{n} e((n+\kappa) z / \lambda)
$$

with 


$$
c_{n}=\sum_{c=-\infty}^{\infty}\left(A_{j}\right) W_{c}(n, \mu) \sum_{m=0}^{\infty} \frac{(-2 \pi i)^{2 m+2}(n+\kappa)^{m+1}\left(\mu+\kappa_{j}\right)^{m}}{c^{2 m+2} \lambda_{j}^{m} \lambda^{m+2} m !(m+1) !} .
$$

Note that if $\alpha=0$ then $c_{0}=0$. By introducing the Bessel function of order 1 of the first kind

$$
J_{1}(z)=\sum_{n=0}^{\infty}(-1)^{n}(z / 2)^{2 n+1} / n !(n+1) !,
$$

we can simplify the coefficients $c_{n}$. We obtain

$$
\begin{aligned}
& c_{n}=\frac{-2 \pi}{2}\left(\frac{\mu+\kappa_{j}}{\lambda_{j}}\right)^{-1 / 2}\left(\frac{n+\kappa}{\lambda}\right)^{1 / 2} \\
& \quad \cdot \sum_{c=-\infty}^{\infty}\left(A_{j}\right) \frac{W_{c}(n, \mu)}{|c|} J_{1}\left(\frac{4 \pi}{|c|} \sqrt{ } \sqrt{\left.\left.\frac{\mu+\kappa_{j}}{\lambda_{j}} \cdot \frac{n+\kappa}{\lambda}\right)\right),}\right.
\end{aligned}
$$

which is the same as (5.4) if summation is restricted to those $c \in \mathscr{C}(A)$, $c>0\left(A=A_{j}\right)$.

In order to prove that $G\left(z, v, A_{j}, \Gamma, \mu\right)$ has at most polar singularities at the cusps we state and prove a lemma which is of interest in its own right.

LEMMA 4. If the Kloosterman sums (5.2) have the estimate $O\left(|c|^{\xi}\right)$ with $1 / 2<\xi<1$, then

$$
G\left(z, v, A_{j}, \Gamma, \mu\right) \mid A_{k}=G\left(z, v_{k}, A_{j} A_{k}, A_{k}^{-1} \Gamma A_{k}, \mu\right)
$$

for $j, k=1,2, \cdots, \sigma$ where $v_{k}$ is the character on $A_{k}^{-1} \Gamma A_{k}$ introduced by $v$ on $\Gamma$. The operator $\mid A_{k}$ is defined in (2.12).

Proof. The assumption on the Kloosterman sums guarantees the existence of the Poincaré series on both sides of (5.8). We remark that $\left(A_{j} A_{k}\right)^{-1} \infty$, $j=1,2, \cdots, \sigma$ is a complete set of inequivalent cusps for $A_{k}^{-1} \Gamma A_{k}$, $\lambda\left(A_{j} A_{k}, A_{k}^{-1} \Gamma A_{k}\right)=\lambda\left(A_{j}, \Gamma\right)$ and that ${ }_{\kappa}\left(A_{j} A_{k}, A_{k}^{-1} \Gamma A_{k}\right)=\kappa\left(A_{j}, \Gamma\right)$. We further note that if $V \in A_{j} \Gamma$ then $V A_{k} \in A_{j} A_{k} \cdot A_{k}^{-1} \Gamma A_{k}$, so that as $(c, d)$ runs over $\mathscr{L}\left(K, A_{j}, \Gamma\right),\left(c^{\prime}, d^{\prime}\right)=(c, d) A_{k}$ runs over $\mathscr{L}\left(K, A_{j} A_{k}, A_{k}^{-1} \Gamma A_{k}\right)$. By Lemma 3

$$
\begin{aligned}
G\left(z, v, A_{j}, \Gamma, \mu\right) \mid A_{k} \\
=\left(c_{k} z+d_{k}\right)^{-2} \lim _{K \rightarrow \infty} \sum_{(c, d) \in Y\left(K, A_{j}, \mathrm{\Gamma}\right)} \bar{v}\left(A_{j}^{-1} V\right)\left(c A_{k} z+d\right)^{-2} e\left(\left(\mu+\kappa_{j}\right) V A_{k} z / \lambda_{j}\right) \\
\quad=\lim _{K \rightarrow \infty} \sum_{(c, d) \in \mathcal{Y}\left(K, A_{j} A_{k}, A_{k}^{-1} \Gamma A_{k}\right)} \bar{v}_{k}\left(\left(A_{j} A_{k}\right)^{-1} V\right)(c z+d)^{-2} e\left(\left(\mu+\kappa_{j}\right) V z / \lambda_{j}\right),
\end{aligned}
$$

which by Lemma 3 and the above remarks is $G\left(z, v_{k}, A_{j} A_{k}, A_{k}^{-1} \Gamma A_{k}, \mu\right)$. This completes the proof.

We finish the proof of Theorem 1 by showing that $G$ has at most polar singularities at the cusps. Lehner $[11$, p. 68$]$ has shown that this is equivalent 
to showing that $G$ possesses a definite limit (finite or infinite) as $z$ approaches the cusp from within the fundamental region. It is clear from (5.3) that this is satisfied at the infinite cusp. Let us consider what happens at $p_{k}=A_{k}^{-1} \infty$, $2 \leqq k \leqq \sigma$. Вy $(5.8), G\left(z, v, A_{j}, \Gamma, \mu\right)=\left(c_{k} z+d_{k}\right)^{-2} G\left(A_{k} z, v_{k}, A_{j} A_{k}^{-1}, A_{k} \Gamma A_{k}^{-1}, \mu\right)$. Now as $z \rightarrow p_{k}, A_{k} z \rightarrow \infty$, so that from the Fourier expansion (5.3) for the Poincaré series $G\left(z, v_{k}, A_{j} A_{k}^{-1}, A_{k} \Gamma A_{k}^{-1}, \mu\right)$ we see that the right-hand side tends exponentially to zero if $j \neq k$, or $j=k$ and $\mu+\kappa>0$, on the other hand if $j=k$ and $\mu<0$ then the Poincaré series tends exponentially to $\infty$. This completes the proof of Theorem 1 .

6. We introduced Kloosterman sums

$$
\begin{aligned}
W_{c}\left(n+\kappa, A_{j}, \Gamma, \mu\right. & \left.+\kappa_{j}\right) \\
& =\sum_{d \in U_{c}\left(A_{j}\right)} \bar{v}\left(A_{j}^{-1} V_{c, d}\right) e\left((n+\kappa) d / c \lambda+\left(\mu+\kappa_{j}\right) a / c \lambda_{j}\right),
\end{aligned}
$$

where $V_{c, d}=(a b \mid c d) \in A_{j} \Gamma$. For these sums we assumed the estimate $O\left(|c|^{\xi}\right), 1 / 2<\xi<1$ for fixed $\mu\left(\mu+\kappa_{j} \neq 0\right)$. In this section we give some examples when this estimate is valid. Our results are based on the following theorem of Petersson [21]: Let $\Gamma$ be a congruence subgroup of the modular group, $v$ a character on $\Gamma$ and suppose there exists an $N$ such that $\Gamma(N) \subset \Gamma$ and $v=1$ on $\Gamma(N)$. Then the Kloosterman sum (6.1) has the estimate $O\left(|c|^{1 / 2+\imath}\right), \epsilon>0$, for fixed $\mu$.

We shall consider either homogeneous groups $\bar{\Gamma}$ and characters $v$ for which $v(-I)=1$ or characters in inhomogeneous groups $\Gamma$. Before we state our proposition, we introduce some new subgroups of the modular group. The congruence group $\Gamma_{0}(n)$ consists of those matrices $v=(a b \mid c d)$ in $\bar{\Gamma}(1)$ for which $c \equiv 0 \bmod n$. The modular group $\Gamma(1)$ has unique normal subgroups of index 2 and index 3 ; let them be denoted by $\Gamma_{2}$ and $\Gamma_{3}$ respectively. We collect our examples in the following proposition.

Proposition. The Kloosterman sum (6.1) has the estimate $O\left(|c|^{1 / 2+\iota}\right)$, $\epsilon>0$ for fixed $\mu$ in the following cases: $\Gamma=$

(a) $\bar{\Gamma}(1)$ and $v$ a character on $\bar{\Gamma}(1)$;

(a) ${ }^{\prime} \bar{\Gamma}$ a congruence group and $v$ a character on $\bar{\Gamma}(1)$;

(b) $\bar{\Gamma}_{0}(q), q \equiv 1 \bmod 4, q$ a prime and $v(V)=v((a b \mid c d))=(d / p)$, the Legendre symbol;

(c) $\Gamma_{2}\left(\right.$ or $\left.\Gamma_{3}\right)$ and $v$ a character on $\Gamma_{2}\left(\right.$ or $\left.\Gamma_{3}\right)$;

(c) ${ }^{\prime} \Gamma$ a congruence group and $v$ a character on $\Gamma_{2}$ or $\Gamma_{3}$.

(a) van Lint [14] proved that $\bar{\Gamma}(12) \subset \bar{\Gamma}^{\prime}(1)$, where $\bar{\Gamma}^{\prime}(1)$ denotes the commutator subgroup of $\bar{\Gamma}(1)$ (a prime on a group shall have this meaning in this paper). More recently, Newman [15] has shown that $\Gamma(6) \subset \Gamma^{\prime}(1)$. Either of these results will be suitable for our purpose. Suppose $v$ is a character on $\bar{\Gamma}(1)$ (which satisfies $v(-I)=1$; hence a character on $\Gamma$ ); then $v=1$ on $\bar{\Gamma}^{\prime}(1) \supset \bar{\Gamma}(12)$. Petersson's theorem applies to show that any 
Kloosterman sum associated with $\bar{\Gamma}(1)$ and $v$ has the estimate $O\left(|c|^{1 / 2+\epsilon}\right)$ ( $\mu$ fixed). This was also obtained by Lehner [10] in another way. However, we can obtain additional dividends. Suppose $v$ is a character on $\bar{\Gamma}(1)$ and $\bar{\Gamma}$ is a congruence subgroup of level $N$ then $\bar{\Gamma} \supset \bar{\Gamma}(12 N)$, and since $\bar{\Gamma}(12)$ $\supset \bar{\Gamma}(12 N), v=1$ on $\bar{\Gamma}(12 N)$. Moreover a transform of $\bar{\Gamma}, B^{-1} \bar{\Gamma} B \supset \bar{\Gamma}(12 N)$ since $\bar{\Gamma}(12 N)$ is normal in $\bar{\Gamma}(1)$. Thus the induced character $v^{\prime}$ on $B^{-1} \bar{\Gamma} B$ will also be 1 on $\bar{\Gamma}(12 N)$. This implies that the Kloosterman sums associated with $v$ and $\bar{\Gamma}$ have the required estimate.

(b) Hecke [6] considered modular forms on the congruence subgroup $\bar{\Gamma}_{0}(q) \supset \bar{\Gamma}(q)$. If $q$ is a prime of the form $4 m+1$ then

$$
v(V)=v((a b \mid c d))=(d / q),
$$

where $(d / q)$ is Legendre's symbol, is a character on $\Gamma_{0}(q)$ for which $v(-I)$ $=1$. We see that $v=1$ on $\bar{\Gamma}(q)$. Petersson's theorem applies to give the estimate (2.16).

(c) Various authors have shown that $\Gamma(6)=\Gamma_{2}^{\prime} \cap \Gamma_{3}^{\prime}$ (see [16] or K. Wohlfahrt, Illinois J. Math. (1964), to appear). This of course implies that the characters of $\Gamma_{2}$ and $\Gamma_{3}$ vanish of $\Gamma(6)$. The groups $\Gamma_{2}$ and $\Gamma_{3}$ have been studied by Newman [15] and Gunning [3]. $\Gamma_{2}$ is generated by 2 elements of order 3 and $\Gamma_{3}$ is generated by 3 elements of order 2 . Thus $\Gamma_{2}$ has 9 characters and $\Gamma_{3}$ has 8 characters. We obtain the extension $(c)^{\prime}$ as in part (a).

7. We should like to show that every form in $\mathscr{C}^{0}(\Gamma,-2, v)$ can be written as a (finite) linear combination of the Poincaré series (3.3) when they are known to exist. This can easily be established by showing that the Poincaré series (3.3) satisfy the "inner product formula" (7.2). In some instances we can even give basis for $\mathscr{L}^{\circ}(\Gamma,-2, v)$ in terms of Poincaré series. These include $\Gamma=\Gamma_{2}, \Gamma_{3}$, and $\Gamma=\Gamma(N)$ for $N=1,2, \cdots, 10,12$. We shall require that $v=1$ for $N \geqq 6$. Once (7.2) is established, these results will be consequences of Petersson's theory ([18], [19], [20]).

Petersson [18] introduced an inner product for automorphic cusp forms of dimension $-r$ which in our case is given by

$$
(f, g)=(f, g ; \Gamma)=\int_{R(\Gamma)} f(z) \overline{g(z)} d x d y,
$$

where $R(\Gamma)$ is a fundamental region for $\Gamma$. The integral is a Lebesgue integral. It is known that for $f, g \in \mathscr{C}^{0}(\Gamma,-2, v)$ the integral converges absolutely and that it is independent of the choice of a fundamental region for $\Gamma$.

Theorem 2. For $\mu+\kappa_{j}>0$ and $F(z) \in \mathscr{C}^{0}(\Gamma, v,-2)$ we have

$$
\left(F, G\left(z, v, A_{j}, \Gamma, \mu\right)\right)=\left[\lambda_{j} / 2\left(\mu+\kappa_{j}\right)\right] a_{\mu}\left(F, A_{j}, \Gamma\right),
$$


where $a_{\mu}\left(F, A_{j}, \Gamma\right)$ is the $\mu$ th Fourier coefficient in the expansion of $F$ at the cusp $\dot{p}_{j}=A_{j}^{-1} \infty$ (see (2.11)).

We shall not give a complete proof of this theorem. When one proceeds formally there is no difficulty in obtaining (7.2), as say, following the proof given in $\left[12\right.$, Chapter VIII, 4c]. Let $\bigcup_{j=1} S_{j}$ be a partition of $R(\Gamma)$ where $S_{j}$ is a neighborhood of the cusp $p_{j}=A_{j}^{-1} \infty, j=1,2, \cdots, \sigma$. Then instead of introducing the double series for $G\left(z, v, A^{-1}, A \Gamma A^{-1}, \mu\right)$ (we have dropped the subscript $j$ ) in the integral

$$
\int_{A S_{j}} G\left(z, v^{\prime}, A^{-1}, A \Gamma A^{-1}, \mu\right) F_{A}(z) d x d y
$$

as is customarily done, we substitute (3.13). We note that the estimate $F_{A}(z)=O\left(e^{-2 \pi \beta y}\right)$ where $\beta=1 / 2$ if $\kappa=0$ and $\beta=\kappa / 2$ if $\kappa>0$ is valid for $z \in A S_{j}$ (see (2.11)). Now using the fact that $H(c, z)=H_{1}(c, z)+H_{2}(c, z)$ and the estimates (3.9) and (3.12) we can justify the interchange of integration and summation (on $c$ ). The next step is to introduce the series (3.2) for $H(c, z)$. It is then easier to justify the interchange of summation on $d$ and integration. We obtain after a change of variables

$$
\lim _{K \rightarrow \infty} \sum_{c \in+\left(A, A \Gamma A^{-1}\right) ;|c|<K} \sum_{d \in\left(x, A, A \Gamma A^{-1}\right)} \int_{v A S} F_{A}(z) e((\mu+\kappa) z / \lambda) d x d y .
$$

From this point the proof given in [12] can be followed.

It is known that a modular form in $\mathscr{C}^{0}(\Gamma,-2, v)$ has a fixed positive number of zeros. This implies that the dimension of the vector space $\mathscr{C}^{0}(\Gamma,-2, v)$ is finite. Then we see that (7.2) implies that the set $G\left(z, A_{j}, \mu\right): \mu+\kappa_{j}>0, \mu$ an integer spans $\mathscr{C}^{0}(\Gamma,-2, v)$ because the only form in $\mathscr{C}^{0}(\Gamma,-2, v)$ orthogonal to all these Poincaré series has all its Fourier coefficients at the cusp $p_{j}=A_{j}^{-1} \infty$ equal to 0 . These results have been known for some time $([18],[19])$. Petersson in 1948 [20] showed that if we set $\nu=\operatorname{dim} \mathscr{C}^{0}(\Gamma,-2, v)$ and $\nu-2 p+1 \geqq 0$ where $p$ is the genus of $\Gamma$ (see [2], [3]) then the Poincaré series $G\left(z, A_{j}, \mu\right): \mu=0,1,2, \cdots, \nu+p-1$ if $\kappa_{j}>0$, or $\mu=1,2, \cdots, \nu+p$ if $\kappa_{j}=0, \operatorname{span} \mathscr{C}^{0}(\Gamma,-2, v)$. He further showed in the same paper that the first $\nu-p$ Poincaré series are linearly independent (that is, $\mu=0,1, \cdots, \nu-p-1$, if $\kappa_{j}>0$ or $\mu=1,2, \cdots, \nu-p$ if $\kappa_{j}=0$ ). The proofs were not for these conditionally convergent Poincaré series, however, they would carry through for any set of functions $\phi(z, \mu)$, $\mu=1,2, \cdots$ which satisfied (7.2). In particular we see then that if the group $\Gamma$ is of genus 0 that the first $\nu$ Poincare series span the space $\mathscr{C}^{0}(\Gamma,-2, v)$. This is the case for $\Gamma(1), \Gamma_{2}, \Gamma_{3}$ and $\Gamma(N), N=1,2, \cdots, 5$ [3]. We shall give the dimension of these vector spaces below.

In 1940 Petersson [18] noted that the smallest system of values for the parameter $\mu, \mu_{1}<\mu_{2}<\cdots<\mu_{\nu}$ for which the Poincaré series $G\left(z, A_{j}, \mu_{k}\right)$, $k=1,2, \cdots, \nu$, span $\mathscr{C}^{0}(\Gamma,-2, v)$ depends upon what he called the 
"Weierstrass character" of the point $A_{j}^{-1} \infty=p_{j}$. He proved that these are exactly $\nu$ natural numbers $0<m_{1}+1<m_{2}+1<\cdots<m_{\nu}+1 \leqq \nu+p$ for which there exists no form in the class $\{\Gamma, 0,1 / v\}$ with a pole of order $m_{k}+1+\kappa_{j}$ at $p_{j}$ and elsewhere regular. The order of the pole is determined by the expansion (2.11). A consequence of this was that the Poincare series with $\mu=m_{k}+\lambda$, where $\lambda=0$ if $\kappa>0, \lambda=1$ if $\kappa=0, k=1,2, \cdots, \nu$ is a basis for $\mathscr{L}^{0}(\Gamma,-2, \nu)$. In view of his later result we see that $m_{1}+1=1$, $m_{2}+1=2, \cdots, m_{\nu-p}+1=\nu-p$.

The problem is to determine which of the numbers $n, \nu-p<n \leqq \nu+p$, are gaps. In the case $v=1$ then $v=p$ and this the problem of determining the Weierstrass gap sequence at the point $A_{j}^{-1} \infty$. Recently this has been done by Joseph Lewittes [13] for the groups $\Gamma(N), N=7,8, \cdots, 10,12$.

(a) For the groups $\Gamma=\Gamma(1)$ and $\Gamma=\Gamma_{2}$ there is only one character for which $\nu=\operatorname{dim} \mathscr{C}^{0}(\Gamma,-2, v)>0$ and in each case $\nu=1$. The dimension of $\mathscr{C}^{0}(\Gamma,-2, v)$ was calculated using Petersson's form of the Riemann-Roch theorem [18] applicable to automorphic forms. In the case of $\Gamma=\Gamma(1)$ it is the character determined by $v(S)=-1, v(U S)=e(-\pi / 3)$ which gives rise to $\nu=1$. For $\Gamma=\Gamma_{2}$ the character determined by $v(U S)=v(S U)$ $=e(-\pi / 3)$ gives $\nu=1$.

(b) In the case of $\Gamma=\Gamma_{0}(q)$ and $v(V)=(d / q)$, the system studied by Hecke [5], Hecke determined the dimension of the space cusp forms. The first case for which $\nu$ is positive is $q=29$ and here it is $\nu=2$.

(c) We turn to the more interesting examples $\Gamma=\Gamma(N)$. We find, using Petersson's form of the Riemann-Roch theorem,

$$
\nu=\operatorname{dim} \mathscr{C}^{0}(\Gamma(N), v,-2)=p(N)-1+\sum_{1}^{o(N)}\left(\left(1-\kappa_{j}\right)\right)+ \begin{cases}0, & v \neq 1, \\ 1, & v=1,\end{cases}
$$

where $((x))=x-[x]$, the fractional part of $x$. The genus of $\Gamma(N)$ is given by

$$
p=p(N)=\left\{\begin{array}{l}
0, \quad 1<N<5, \\
1+\frac{N^{2}(N-6)}{24} \prod_{q \mid N}\left(1-\frac{1}{q^{2}}\right), \quad n>2,
\end{array}\right.
$$

and

$$
\sigma=\sigma(N)=\left\{\begin{array}{l}
3, \quad N=2, \\
\frac{N^{2}}{2} \prod_{q \mid N}\left(1-\frac{1}{q^{2}}\right), \quad n>2,
\end{array}\right.
$$

where the product is extended over all prime divisors $q$ of $N$.

Consider the case

(i) $\Gamma=\Gamma(5)$. Then depending upon $v$ we see that $0<\nu<\sigma(S)-1=11$. Petersson [19] shows that $\sum_{i}^{i} \kappa_{j} \equiv 0 \bmod 1$ is a requirement of a character. For example, if $v\left(A_{j} U^{5} A_{j}^{-1}\right)=e(1 / 4)$ then $\nu=8$ and since $p(5)=0$ the 
Poincaré series $G(z, I, \mu), \mu=0,1,2, \cdots, 7$ span $\mathscr{C}^{0}(\Gamma(5),-2, v)$.

(ii) Let $\Gamma=\Gamma(N), N=7,8,9,10,12$ and $v=1$. Lewittes [13] has determined the Weierstrass gap sequence at $\infty$, hence at $A_{j}^{-1} \infty$ since $\Gamma(N)$ is normal in $\Gamma(1)$, for those values at $N$. We see that in this case that $\nu=p(N)$. For, say, $N=10, p(N)=13$, and the gaps are $\mu=1,2, \cdots, 9,11,13,17,19$. Hence the Poincaré series $G\left(z, A_{j}, \mu\right), \mu=1,2, \ldots, 9,11,13,17,19$, span $\mathscr{C}^{0}(\Gamma(10),-2,1)$.

\section{REFERENCES}

1. L. E. Dickson, Studies in the theory of numbers, Univ. Chicago Press, Chicago, Ill., 1930.

2. L. R. Ford, Automorphic functions, 2nd ed., Chelsea, New York, 1951.

3. R. C. Gunning, Lectures on modular forms, Annals of Mathematics Studies No. 48, Princeton Univ. Press, Princeton, N. J., 1962.

4. E. Hecke, Theorie der Eisensteinschen Reihen hoherer Stufe und ihre Andwendung auf Funktionentheorie und Arithmetik, Abh. Math. Sem. Univ. Hamburg 5 (1927), 199-224.

5. , Analytische Arithmetik der positiven quadratischen Formen, Danske-Vid. Selsk.

Math.-Fys. Medd. 17 (1940), no. 12.

6. Konrad Knopp, Theory of functions. II. Applications and continuation of the general theory, Dover, New York, 1947.

7. M. I. Knopp, Fourier series of automorphic forms of non-negative dimension, Illinois J. Math. 5 (1961), 18-42.

8. _ Automorphic forms of non-negative dimension and exponential sums, Michigan Math. J. 7 (1960), 257-287.

9. A. G. Kurosh, The theory of groups, 2nd ed., Vol. 2, Chelsea, New York, 1960.

10. J. Lehner, On modular forms of negative dimension, Michigan Math. J. 6 (1959), 71-88.

11.

Math. J. 7 (1960), 65-74.

12. , Discontinuous groups and automorphic functions, Math. Surveys No. 8, Amer.

Math. Soc., Providence, R. I., 1964.

13. J. Lewittes, Gaps at Weierstrass points for the modular group, Bull. Amer. Math. Soc. 69 (1963), 578-582.

14. J. H. van Lint, On the multiplier system of the Riemann-Dedekind function $\eta(\tau)$, Nederl. Akad. Wetensch. Proc. Ser. A 20 (1958), 522-527.

15. M. Newman, The structure of some subgroups of the modular group, Illinois J. Math. 6 (1962), 480-487.

16. M. Newman and J. R. Smart, Note on a subgroup of the modular group, Proc. Amer. Math. Soc. 14 (1963), 102-104.

17. H. Petersson, Zur analytischen Theorie der Grenzkreisgruppen II, Math. Ann. 115 (1938), 175-204.

18. Uber eine Metrisierung der automorphen Formen und die Theorie der Poincareschen Reihen, Math. Ann. 117 (1940), 453-537.

19. _ Automorphe Formen als metrische Invarianten. I, II, Math. Nachr. 1 (1948), 158-212, 218-257.

20. U Uber Weierstrasspunkte und die expliziten Darstellunger der automorphen Formen von reeller Dimension, Math. Z. 52 (1949), 32-59.

21. U Uber Modulfunktionen und Partitionenprobleme, Abh. Deutsch. Akad. Wiss. Berlin. Kl. Math. Allg. Nat. 1954, no. 2.

22. __ Explizite Konstruktion der automorphen Orthogonalfunktionen in den multiplikativen Differentialklassen, Math. Nachr. 16 (1957), 343-368.

23. H. Rademacher, The Fourier series and the functional equation of the absolute modular invariant $J(\tau)$, Amer. J. Math. 61 (1939), 237-248.

UNIVERSITY OF WISCONSIN,

Madison, Wisconsin 